\title{
Nonlinear Image Enhancement by Self-Adaptive Sigmoid Function
}

\author{
Qihang He, Congyao Zhang and Dong C. Liu \\ College of computer science, Sichuan University, Sichuan China, 610065 \\ scu_heqihang@163.com
}

\begin{abstract}
Nonlinear imaging is more sensitive to changes in components, structure and pathological state of a tissue in comparison with the traditional ultrasound imaging. However, in view of the particularity of nonlinear imaging, the contrast of an image is generally very low and thus a doctor cannot clearly distinguish distribution of nonlinear parameters clearly. Therefore, the paper aims to design a self-adaptive sigmoid function to enhance the original nonlinear image. First of all, we needed to find an interesting area which included the different nonlinear acoustic parameters according to harmonic energy. Next, then the histogram of ROI was analyzed to obtain two parameters of sigmoid function values, and the contrast of the image was increased prominently by this method. At last, the contrast to noise ratio (CNR) was calculated to prove that the contrast of the image which was enhanced by this method improved by 5 times in comparison with the raw nonlinear image.
\end{abstract}

Keywords: Nonlinear imaging; Enhancement; Histogram; Sigmoid; CNR

\section{Introduction}

The method for measuring the nonlinear parameter of tissue and imaging in an ultrasound system becomes the hotspot in the field of nonlinear acoustics [1]. Actually, in comparison with the traditional ultrasound imaging which can reflect the acoustic impedance of the tissue, speed of sound, sound attenuation and other linear parameters, the nonlinear acoustic parameter is more sensitive to changes in the tissue's components, structure and pathological state[3-4]. Hence, the new ultrasound tissue characterization method for nonlinearity parameter measurement and imaging plays a significant role in medical applications.

There are many methods used for nonlinearity parameter measurement and imaging, among which the thermodynamic method is classical [5]. Measurement of pulse-echo single-frequency acoustic nonlinearity parameters was put forward [6], and then the finite amplitude insert substitution (FAIS) method was proposed [7]. The direct measurement in reflection mode was put forward after that [8]. However, in view of the particular requirements for laboratory equipment and environment, these methods cannot be used in medical ultrasound imaging systems with the normal medical probe. The nonlinearity imaging method based on a medical ultrasound imaging system and a regular linear array probe was put forward in 2014 [9]. As shown in Figure 1, the result of this nonlinear image is able to effectively image the distribution of nonlinear acoustic parameters inside the biological tissue. However, the image is inadequate in contrast, and it is not conducive to visual diagnosis of a doctor.

Therefore, the paper aims to design a self-adaptive sigmoid function to enhance the nonlinear image. The above method which is used to collect the experimental data will be proposed in Section 2. The interesting area in our nonlinear image will be proposed in Section 3. The adaptive sigmoid function will be offered in Section 4, wherein the regular sigmoid function will be described while parameters of this function will be discussed. 
Next, qualitative comparison and quantitative analysis of the contrast to noise ratio (CNR) will be achieved. In the end, the paper will make a conclusion in Section 5.

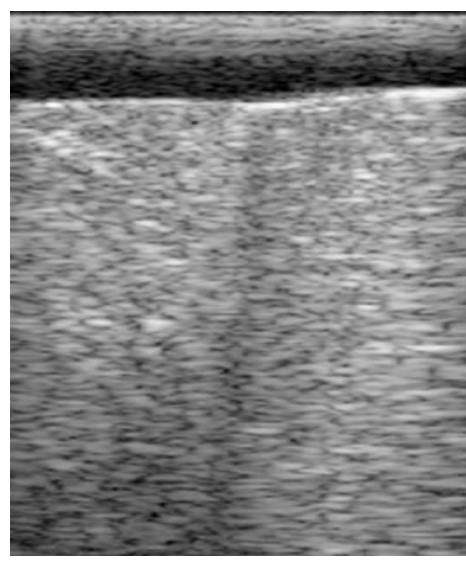

(a). B-mode image

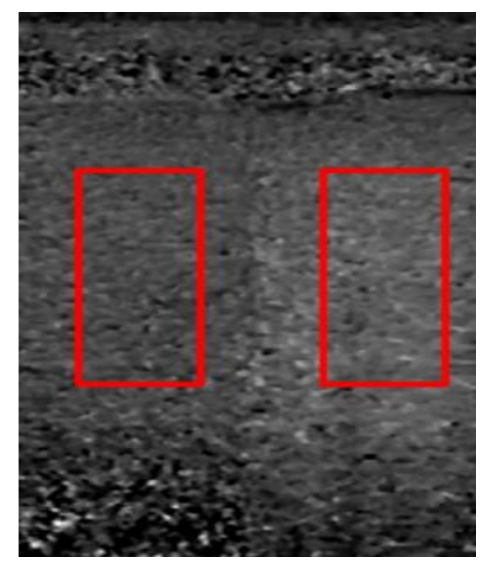

(b). nonlinear image

Figure 1. Contrast of Two Image

\section{Experimental Data Acquisition}

The experimental data was collected on iMago C21 as shown in Figure 2 which was developed by the Saset (ChengDu) Inc.

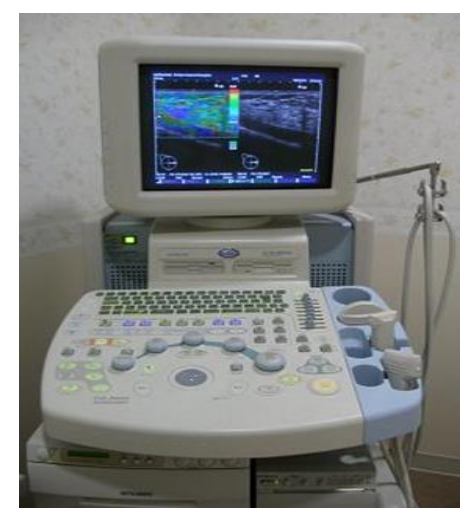

Figure 2. iMago C21

In this experiment, an agar phantom with fresh porcine tissue inside as shown in Figure 3 , which the left part is the porcine muscle and the right part is the porcine fat, is applied to investigate the performance.



Figure 3. The Fresh Porcine Tissue 
As the main method which can be used on iMago C21 was given [10]. First two ultrasonic pulses at different excitation voltage were transmited, then two B-mode images were presented in Figure 4, finally the result was the image with high excitation voltage minus the image with low excitation voltage, thus is the nonlinear image as shown in Figure 5 which the left part is the porcine muscle and the right part is the porcine fat.

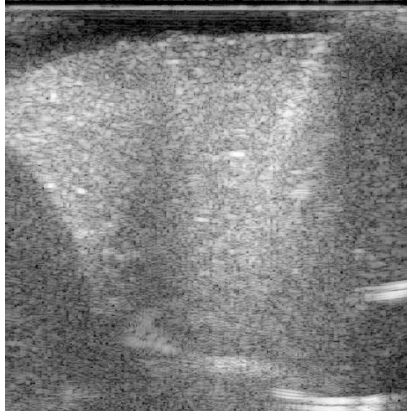

(a) low voltage B-mode

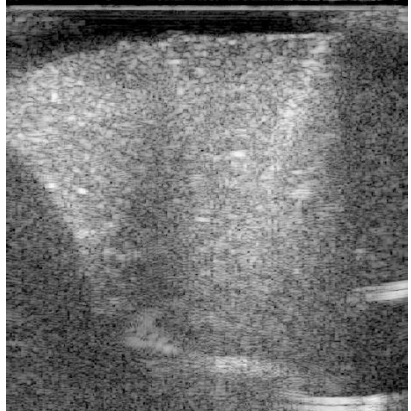

(b) high voltage B-mode

Figure 4. B-Mode Image

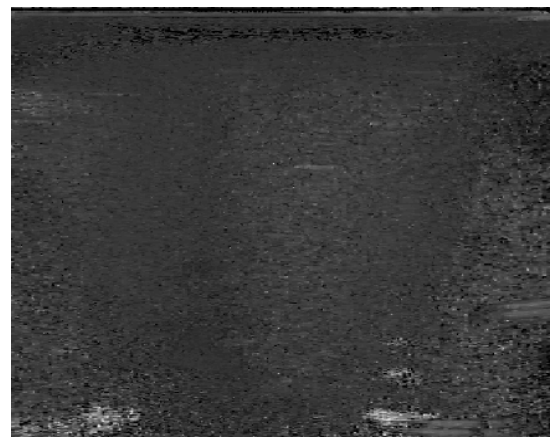

Figure 5. Raw Nonlinear Image

Through experimental results, it is clearly that the image in B-mode unable to distinguish fat and muscle completely, but the nonlinear image can do it. As mentioned in introduction, the resolution of the nonlinear image is very low, and this image cannot be diagnosed by a doctor well. So the next work of this paper is to improve the contrast ratio of this image.

\section{Region of Interest}

With the fundamental frequency of the wave is depleted and higher harmonics are generated, but with the depth of the wave propagation is increased and faster harmonics are attenuated [12]. So the main energy of harmonics concentrate in the mid-field. As Figure 6 shows the human liver tissue with fundamental imaging and tissue harmonic imaging (THI), and the fundamental frequency of ultrasound probe is $2.5 \mathrm{MHz}$. After careful comparison, it is clearly that the resolution of second harmonic image in the mid-field was significantly higher than that of the fundamental picture, and the more detail from the physiological and anatomical structure of the organization can be observed. While in the far-field, due to the faster harmonic attenuation, leading to the rapid decline in image quality, rather than the fundamental image [10]. 


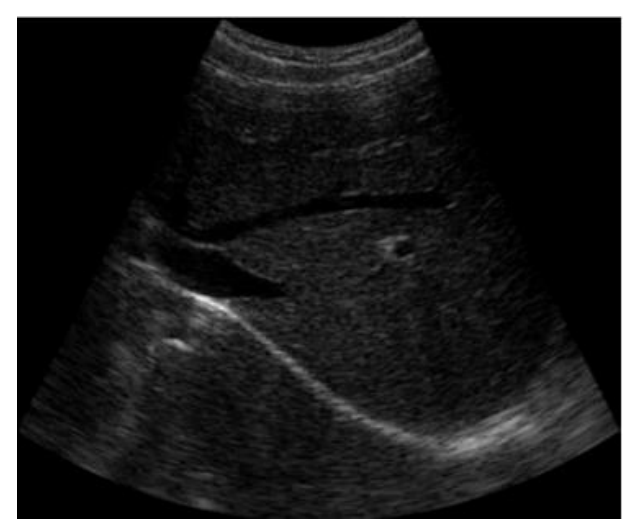

(a)

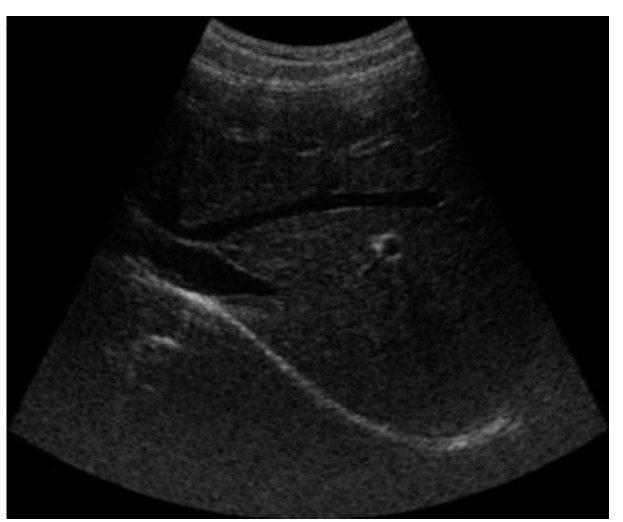

(b)

Figure 6. Human Liver Tissue, (a) is Fundamental Image and (b) is Harmonic Image

For the above reasons, only the data in mid-field is the most meaningful, so in this paper, the region of interest (ROI) is the red rectangle at the mid-field of nonlinear image as shown in Figure 7, which the left part is muscle and the right part is fat.

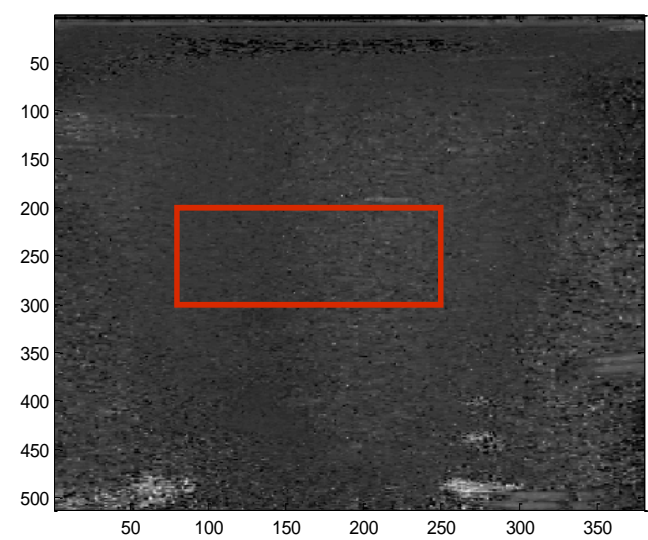

Figure 7: ROI of Nonlinear Image

\section{Self-Adaptive Sigmoid Function}

It is clearly that this image is blurred and the contrast ratio is very low from Figure 5. So it is need to enhance the image so that doctors can easily distinguish the fat and muscle. Common image enhancement algorithm is linear, but generally human visual adaptation considered has nonlinear characteristics of the S-type. So in this paper, a sigmoid function is used for image enhancement adaptively.

Sigmoid function is a continuous nonlinear function, as follows [11]:

$S(x)=\frac{1}{1+e^{(-A *(L(x)-C))}}$

The $A$ controls the degree of the linear gradient shown as Figure 8, and $C$ controls the position of enhancement shown as Figure 9: 


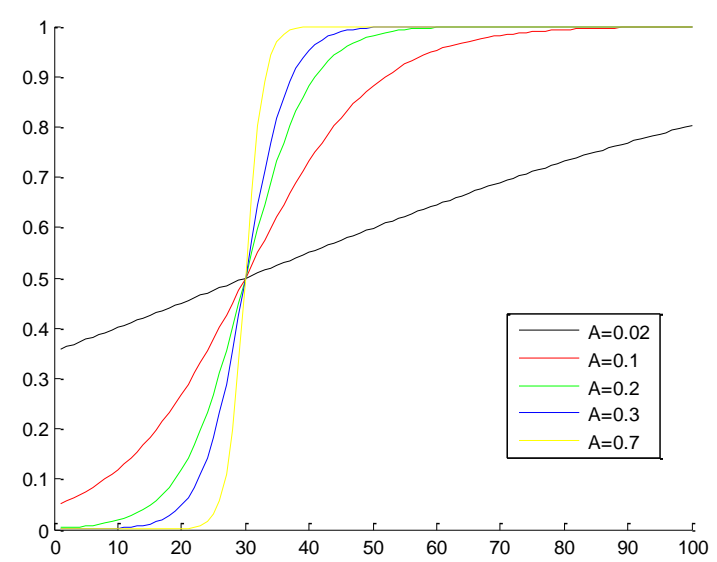

Figure 8: The Curves when a Changed and $\mathrm{C}$ is $\mathbf{3 0}$



Figure 9: The Curves when $C$ Changed and $A$ is 0.1

So depending on the impact of the parameters $\mathrm{A}$ and $\mathrm{C}$ on image enhancement, combined with nonlinear imaging characteristics, we propose an adaptive method to determine the $\mathrm{A}$ and $\mathrm{C}$ of sigmoid function.

\subsection{Method}

Because the global histogram of image contains the values of background and it is a unimodal as Figure 10 shown. So this chart cannot compute the value of $\mathrm{A}$ and $\mathrm{C}$. However, only the value in ROI is meaningful as mentioned in Section 3, so we can compute the histogram of ROI as Figure 11 shown. 


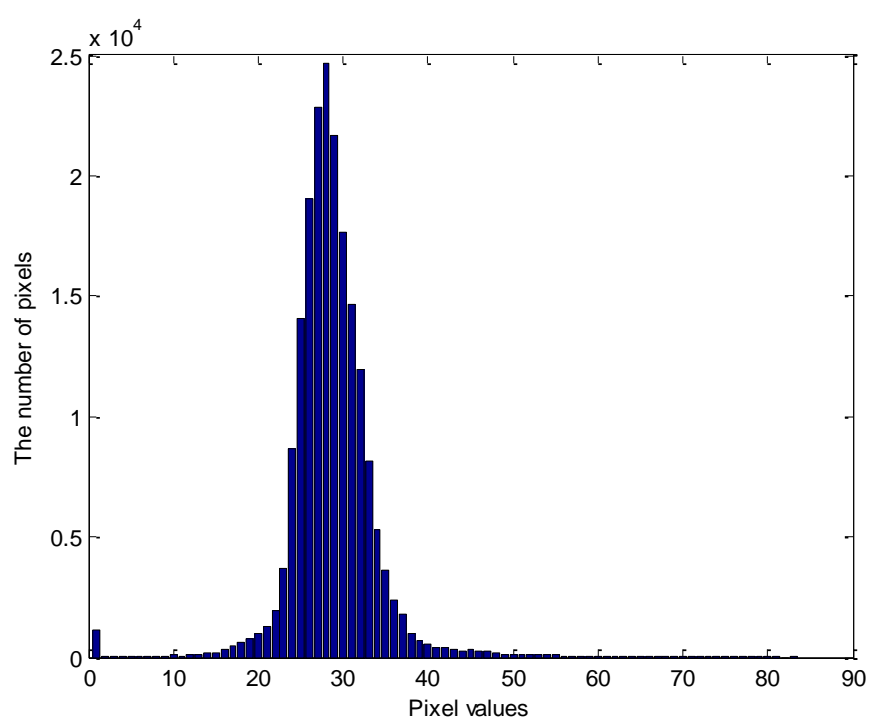

Figure 10. Global Histogram of Image

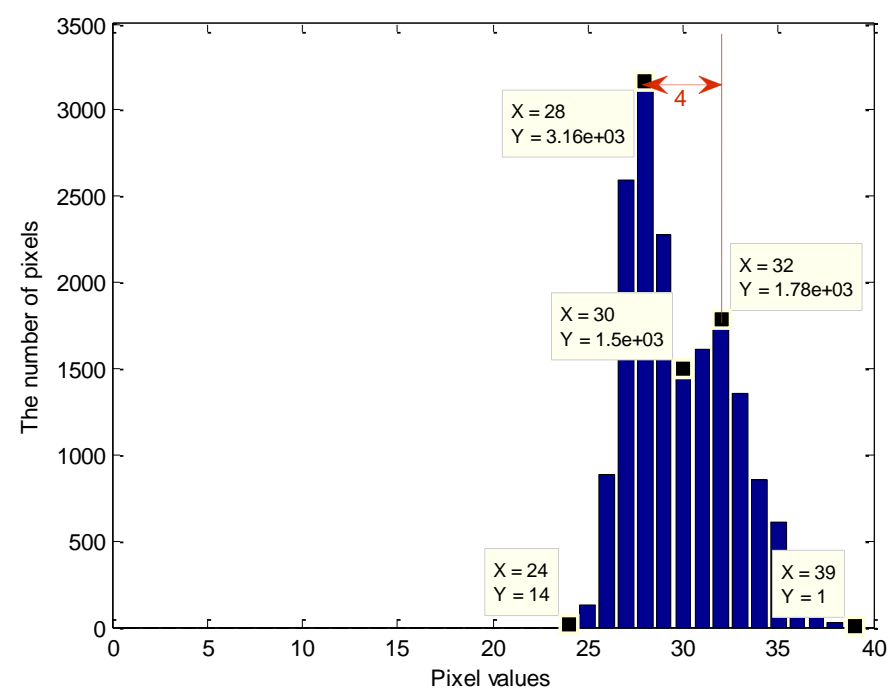

Figure 11. Histogram of ROI

As shown in Figure 11, the peak value represents the fat and muscle, the trough value represents the threshold which to distinguish the fat and muscle. When Parameter $C$ takes different values the position of enhancement is different, thus if $C$ takes a small value is to enhance the pixels in a dark region while if $C$ takes a larger value is to enhance the pixels in a bright region. Because the trough value is special as mentioned above. So if we take $C$ as the trough value, we can separate the fat and muscle clearly. Finally $C$ takes 30 in this paper.

Figure 12 is the derivative of Figure 8, so these values represent slope of corresponding point in Figure 8, and the peak value represents the degree of the linear gradient. Because the slope of the curve on both sides approaching 0, if the slope of a point in Figure 8 is very small then the degree of enhancement is very low, thus the point is not enhanced. So in order to make the value which represent the muscle and fat enhanced, it is must to make sure the slope of the point in Figure 8 which represent that value greater than 0 . Here we define the slope is smaller than the value of 0.2 is equal to 0 . So the range of our 
enhancement is between the two points approaching 0.2 on the both sides of the peak as shown in Figure 12. From Figure 11 we can see the value of the fat and muscle are from 24 to 39, combined with the Figure 12, we can find this range correspond to the curve which $A$ is 0.7 . Finally $A$ takes 0.7 in our paper.



Figure 12. Derivative of Sigmoid Curves

\subsection{Results and Discussion}

The result of enhancement is shown in Figure 13(c), where the parameter $A$ is 0.7 and the parameter $C$ is 30 . Then color mapping was added in ROI to make doctor distinguish tissue easy as shown in Figure 13(d).



(a) B-mode image

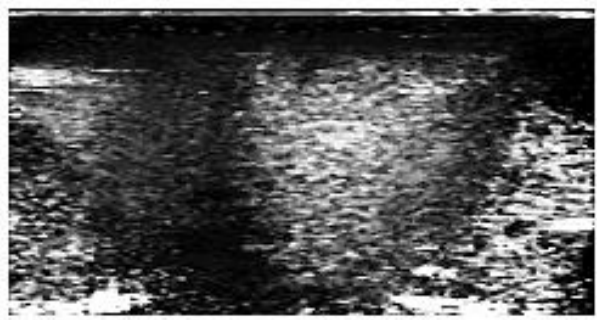

(c) sigmoid enhancement



(b) raw nonlinear image

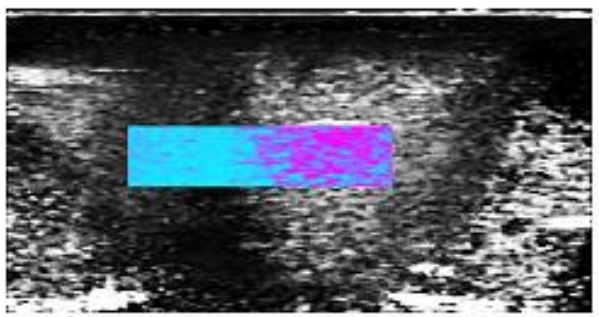

(d) color transparency

Figure 13. Sigmoid Function Adaptive Image Enhancement

As shown in Figure 13 we can see the contrast ratio of the image after sigmoid enhancement is much higher than the raw nonlinear image. Contrast to noise ratio (CNR) is often used to evaluate the quality of image enhancement. As mentioned in Section 2, the ROI at the mid-field so the two region which to compute the CNR at the mid-field too as shown in Figure 14. 




\section{Figure 16. Two Region to Compute the CNR}

Where, the blue rectangle represent the muscle and the red rectangle is the fat, their CNR of each image were computed in Table 2.

Table 2: The CNR of Each Image

\begin{tabular}{|c|c|}
\hline Image & CNR \\
\hline B-mode image & 0.5725 \\
\hline Raw nonlinear image & 1.3638 \\
\hline Sigmoid enhancement & 8.3744 \\
\hline
\end{tabular}

From Table 2, the CNR of the image by the Self-adaptive sigmoid function increased $514.04 \%$ than that of raw nonlinear image. So the self-adaptive sigmoid function improved contrast significantly.

\section{Conclusion}

In this paper, we presented a self-adaptive sigmoid function to enhance the raw nonlinear image. The histogram of ROI determine the two parameters of the sigmoid function to optimize the enhancement, and compared with the raw nonlinear image the contrast improved 5 times. Through this method a doctor can distinguish the tissue with different nonlinear parameters from the nonlinear image clearly.

On the other hand, the ROI determines the sigmoid parameters as also the effect of the image enhancement, so the ROI is very important. In this paper ROI is determined artificially, so we need to determine the ROI based on outline and energy automatically in our further work.

\section{Acknowledgments}

This work was supported in part by the Foundation of China and Natural Science Foundation of Sichuan Province (Grant No. 2013GZX0147-3) and Crossing Research Project (13H0292).

\section{References}

[1] G. Xiu-Fen and Z. Dong, "Acoustic nonlinearity parameter imaging and its application in medical diagnosis", Applied acoustics, vol. 24, no. 4, (2005), pp. 208-215.

[2] Haralick R M, Shanmugam K and Dinstein I H, “Textural features for image classification”, Systems, Man and Cybernetics, IEEE Transactions on, vol. 6, pp. 610-621, (1973). 
[3] L. Xiaozhou, G. Xiufen, R. Bing and Z. Lianzhi, "Ultrasonic characterization for biopathological tissue by using acoustic nonlinearity parameter", Chinese journal of biomedical engineering, vol. 16, (1997), no. 2, pp. 181-185.

[4] Sehgal C M, Bahn R C and Greenleaf J F, "Measurement of the acoustic nonlinearity parameter B/A in human tissues by a thermodynamic method", The Journal of the Acoustical Society of America, vol. 76, no. 4, (1984), pp. 1023-1029.

[5] Zhu Z, Roos M S and Cobb W N, "Determination of the acoustic nonlinearity parameter B/A from phase measurements", The Journal of the Acoustical Society of America, vol. 74, no. 5, (1983), pp. 1518-1521.

[6] Nikoonahad M and Liu D C, "Pulse-echo single frequency acoustic nonlinearity parameter (B/A) measurement", Ultrasonics, Ferroelectrics, and Frequency Control, IEEE Transactions on, vol. 37, no. 3, (1990), pp. 127-134.

[7] Zhang D and Gong X F, "Experimental investigation of the acoustic nonlinearity parameter tomography for excised pathological biological tissues", Ultrasound in medicine \& biology, vol. 25, no. 4, (1999), pp. 593-599.

[8] Harris G R, Liu Y and Maruvada S, "P4H-1 Finite Amplitude Method for Measurement of Nonlinearity Parameter B/A Using Plane-Wave Tone Bursts", In IEEE Ultrasonics Symposium, (2007), pp. 2072-2074.

[9] Gong X F, Zhang D, and Liu J H, "Study of acoustic nonlinearity parameter imaging methods in reflection mode for biological tissues", J Acoust Soc Am, vol. 116, (2004), pp. 1819-1825.

[10] Zhang Congyao, "Research of Method and Technology of Ultrasound Elastography and onlinearity Imaging", Sichuan University, (2014).

[11] Liu L-m, Wang Y-q, Sun Y, Yang D-q and Zhao Y, "Sigmoid function adaptive X-ray image enhancement based on image median", Journal of China Clinic Medical Imaging, DOI:10.3969/j.issn.1008-1062.2011.01.024, vol. 22, no. 1, (2011), pp. 65-67.

[12] Fatemi M and Greenleaf J F, "Real-time assessment of the parameter of nonlinearity in tissue using 'nonlinear shadowing', Ultrasound in medicine \& biology, vol. 22, no. 9, (1996), pp. 1215-1228. 
International Journal of Signal Processing, Image Processing and Pattern Recognition Vol.8, No.11 (2015) 